\title{
Automation and Machine Learning in Transforming the Financial Industry
}

\section{Praveen Kumar Donepudi}

Principal Architect, IT Infrastructure Services, Cognizant Technology Solutions, United States

https://doi.org/10.18034/abr.v9i3.494

\begin{abstract}
The major purpose of this study was to analyze the influence of machine learning on the digital age, particularly in the field of finance. This study involves the application of machine learning, its challenges, opportunities and effect on job openings and operations. This paper is based on the findings of a qualitative study of the text on the subject of machine learning in finance. The theoretical portion of this paper explores the universal framework, such as the past, existing and the next level of the machine learning, with emphasis on its advantages and drawbacks. The study also examines the global recognition of machine learning in the review of artificially intelligent development and startups in European countries. The research methodology used in this study was the evaluation of the qualitative methods in the paper. The study also reviewed twenty electronic records and articles on machine learning in finance. During the research on how computer technology transforms the banking sector, the implementation and impact of artificial intelligence in financing was discussed. Research shows that several financial institutions have significantly benefited from the introduction of a variety of machine learning and artificial intelligence. This paper demonstrates that there is a lack of experience in the field of machine learning, even as many unskilled or semi-qualified tasks carried out by individuals are carried out by machines. This study has shown that, through banking and financial valuation, whether it is manufacturing, data analysis or continuing to invest, there will be many more developments that can get the job done.
\end{abstract}

Key words: Artificial intelligence, Innovation, financial services, virtual assistant, machine learning, technologies

\section{INTRODUCTION}

Study in the fields of software engineering and analytics has been stepped up to develop innovative techniques to obtain information from a wide range of data sets. Statistical information could be of different forms, of different kinds and of comparable quality. Such strategies may leverage the potential of machines to perform tasks such as image recognition and the use of natural scripts through experiential learning. The use of computational methods to solve tasks that historically involve human creativity is commonly referred to as 'artificial intelligence' (AI) (Chui et al., 2016). AI has been in existence as a sector for several years. However, recent improvements in computer technology, combined with advances in information availability and volume, have led to a massive increase in future AI technologies. These technologies are used to detect conditions, decode languages and drive vehicles, and have also been progressively used by the finance industry.

$\mathrm{AI}$ is considered to be the tendency of machines to make sensible brain-like choices and to develop over time. On the other hand, Machine Learning (ML) includes the design of concepts, often statistical models that provide accuracy of predictions and can be created. A number of people associate AI with robots that mimic human functions and creativity in their operation, so the entertainment industries have run their time to convince people that AI applies to robotics (Kokina \& Davenport, 2017). Although this is true, there is more to it through facial recognition software, fingerprint scanners, chat bots, pattern recognition, computational business strategies, and psychological analysis. Initially, the integration of AI in software development was only feasible for large organizations with the ability to employ highly qualified professionals. With time, high- 
complexity AI programs have been designed, with few programming lines in each preferred scripting language; an intelligent system is being designed.

The goal of this article is therefore to examine how and why AI has been applied to various sectors, especially in the context of finance. In addition, the paper answers the research question in the study: what are the risks and opportunities of AI? What are AI's applications for the financial sector? How is AI adopted internationally? What influence would AI have on the career path of finance jobs and mechanisms? How could the development of Ai look like that? Which measures are taken by companies to thrive in a dynamic technological age? A text study of the qualitative research process has been used as an alternative to addressing these issues.

Many modern mobile devices will identify faces and signatures and use them as a primary security check. AI integration has also been used to identify fraud in the financial system, where suspicious transactions are identified and alarms are raised. Several applications have now been developed in the corporate world using AI to create computational models that can be significantly improved (De Prado, 2018). Predictive models may be incorporated into property price forecasts, foreign exchange rates, accounting and finance models, demand forecast models and, to some degree, a nostalgic analysis of how customers respond to a particular product. For example, its incorporation into the corporate world has led to an increase in sales. Amazon, for example, reported more than 60 per cent of its recommendation systems revenues. With the rise in online clientele from large companies, the swapping of human intervention with virtual assistants has become a huge success, with chat bots substituting human labor by offering digital and realtime help.

The financial services sector integrates AI to analyze massive data, track scams by detecting suspicious transactions, interact electronically with clients, and explore a number of other key functions. As far as facial reconfiguration, voice identification and ML are concerned, there are extremely important beneficial events. New technologies deliver great benefits in order to maximize the value of the customer's proposals to drive productivity and efficiency in the enterprise. AI tools will bring significant benefits to the financial sector, enabling certain activities to be automated and improving analytical capabilities compared to conventional techniques that are rapidly becoming obsolete (Chui et al., 2015). However, despite the life-changing advantages of AI applications, they often have different drawbacks that could make them not ideal for carrying out certain tasks, along with a variety of risks that need to be addressed accordingly. This paper demonstrates how the AIlearning machine is used for problem-solving in a series of events that leverages independently and automatically with no human involvement.

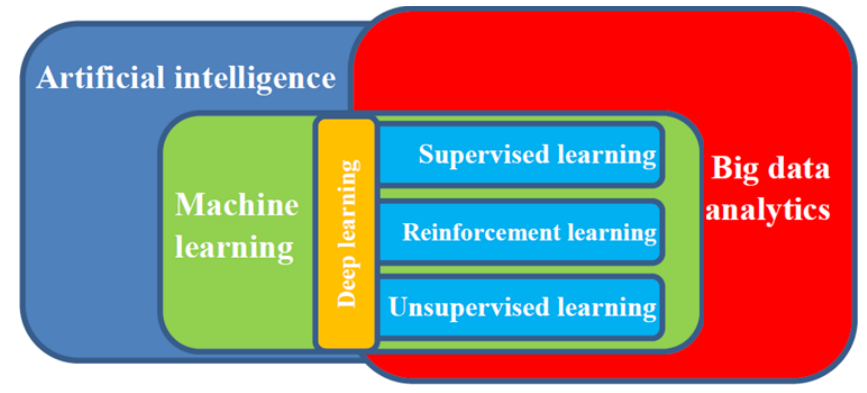

Figure 1: Huge data and the AI in machine learning and analysis

\section{Methodology}

\section{Research Processes}

Analysis includes finding and trying to redefine concerns, designing models or recommended solutions; collecting, arranging and analyzing data; rationalizing and drawing conclusions; and, ultimately, checking the conclusions to determine whether they are appropriate to the assumption. The research methodology describes the research road map and defines the key tasks of the local research community. The aim of this research is to recognize the influence of AI on the financial services sector.

\section{Research Methodology}

This research utilizes qualitative research methodology in studying the application and effects of the $\mathrm{AI}$ in finance besides investigating how artificial intelligence is changing the financial service industry. This research methodology seeks to explore the AI in finance in a broader and detailed way. Qualitative research focuses on quality causes, i.e. phenomena that respond to or involve quality. Qualitative analysis is intended to evaluate such processes or behaviors and then to show how they can be combined and grouped in order to produce measurable results. This form of analysis is more selective and requires a thorough interpretation of the variables. The benefit of this set of methods is that it is versatile and easy to respond to changes throughout the research setting and also at a low cost. Although quantitative data are established, qualitative data will be provided.

\section{Document analysis}

Document analysis is a structured method in which records that can be both written and electronic (computer-based and online world) can be checked or reviewed. The choice of 'document review' as a study tool may have many advantages: it allows the investigator to reach out to 'inaccessible individuals or subjects.' Several documents in the 'public domain' are compiled by professionals and provide very useful knowledge and observations and documentaries that are available to the highest expectations and are therefore very economical. In particular, these records may be an incredibly accurate source of data as they are produced for domestic or foreign reasons. 
For this study, qualitative data-collecting system review was chosen, as it provides an opportunity to analyze the data collected by professionals, who are often not accessible through an outside methodology, and these documents are also quite available. For instance, findings from different international organizations focus on longterm surveys where one scholar cannot perform. These types of documents have therefore been useful for this research, and most of them can be accessed through official websites at the organizational level.

\section{Materials used}

As in every empiric approach used in qualitative research, the study of documents requires to be reviewed and evaluated in order to assess the context, gather insights and obtain information. This paper examines a variety of online resources selected on the basis of the following metrics: "AI and finance" "AI effects in finance," "AI application in finance," "Future of finance" and "AI strategy priorities." Throughout the study, it has been considered that the papers and articles used for the analysis have been accurate.

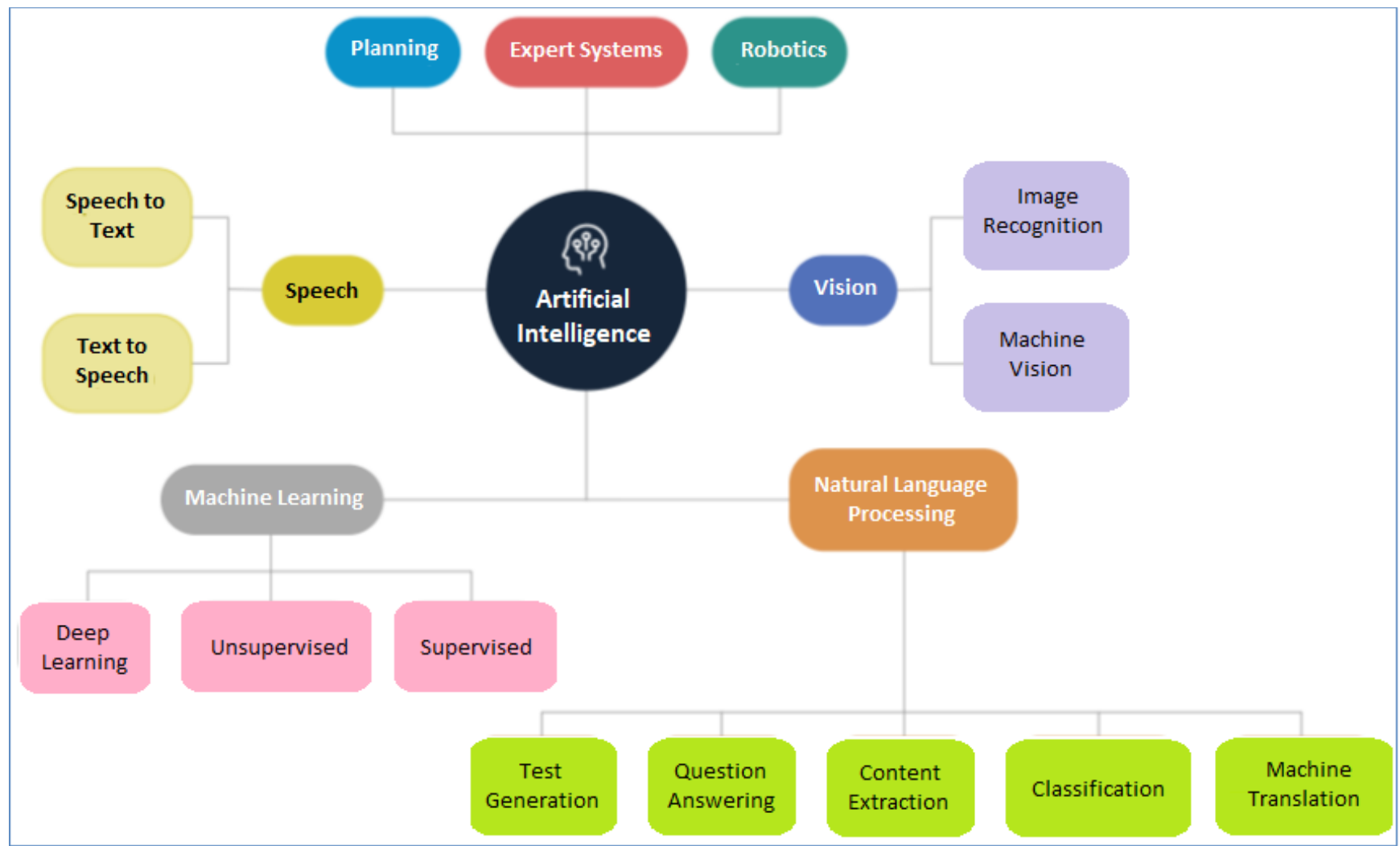

Figure 2: Sub-sections of the AI (Mackenzie, 2015)

AI study includes thinking, representation of information, preparation, learning, NLP, understanding and ability to control and transfer information. General intelligence is one of AI's long-term priorities. Strategies typically include analytical strategies, artificial intelligence, and traditional symbolic AIs. Several mechanisms have been

\section{ARTIFICIAL INTELLIGENCE (Al)}

AI is a broad term that refers to technological advancements that actually make computers "intelligent." There are many different terms related to AI, such as deep learning, ML, image recognition, natural language processing (NLP), cognitive technologies, cognitive enhancement, machine intelligence and increased intelligence. AI, as it is used here, involves every idea of an event. AI is working to create an autonomous, intelligent framework. ML is an AI category that allows the computer to advance its comprehension electronically without direct human intervention (Mullainathan \& Spiess, 2017). There are two techniques that AI operates, one of which is conceptual, while the other focuses on results. In the case of a database system called ML, we should transmit a bunch of data to the system before it can be understood. Machines will learn a lot more about measurements. Computer monitors a lot of highdimensional data and evaluates patterns. When these models have been studied, they will make predictions that individuals have not been able to handle them. The AI comprise of several subfields as illustrated by figure 2 . 
gain meaningful insights that vary from the vast datasets (De Prado, 2018). Information may be categorized into different categories, or information may be configured or misconfigured. Through using techniques, it is possible to maximize the capacity of machines to perform tasks through experiential education, such as NLP and image identification.

AI is gradually developing in a number of different sectors. There are endless requests for AI. Innovation could be applied in a wide range of sectors and businesses. For example, in the medical field, AI has been tested and applied for dosing and separate treatments in patients and surgery. In addition, AI is used in the identification and marking of banking and finance operations, such as the unusual use of ATM cards and large bank deposits, all of which help the fraud unit of the bank (Gomber et al., 2018). AI implementations are also used to streamline and promote business. This can be accomplished through promoting the assessment of production, demand and cost of shares.

\section{Types of artificial intelligence}

Despite the fact that AI is certainly multidimensional, there still are unique types of AI wherein expanded classes fall. There are several concepts and meanings in AI which make it difficult to analyze the distinction among types, subsections, or AI diversity, but each of those has distinct views. A few more AI subsections include ML, Big Data and NLP (Sharma et al., 2014). Nevertheless, four major categories of $\mathrm{AI}$ are discussed in this section: reactive computers, limited memory, consciousness theory, as well as self-awareness.

\section{TYPES OF AI}

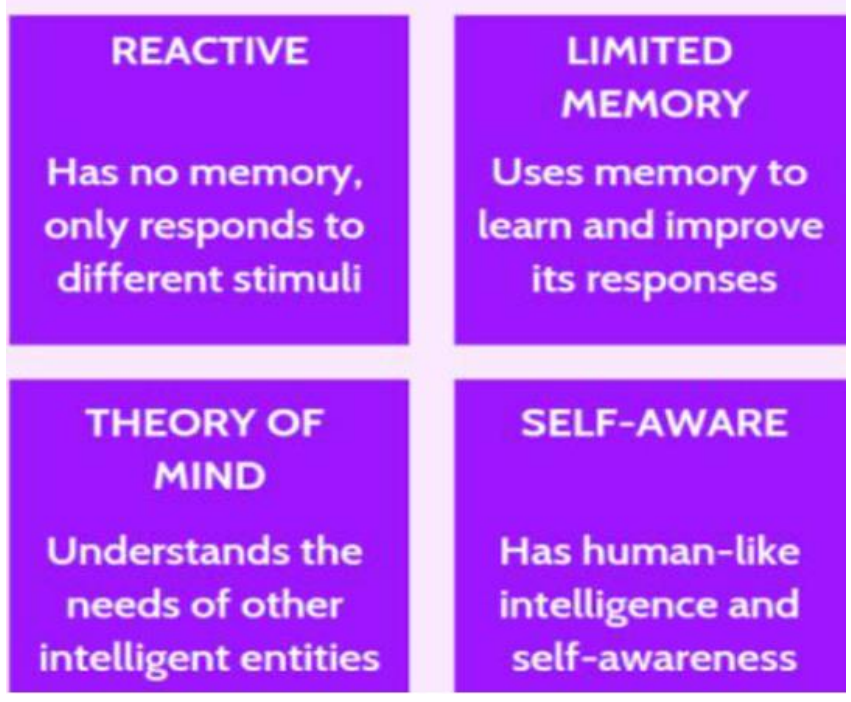

Figure 2: AI types

Reactive machines are important because they cannot retain 'memories' and use previous experience to identify future projects. They're essentially looking at the world, and then they're responding to it. IBM's Deep Blue, performed by chess Grandmaster Kasporov, is a sensitive machine that recognizes and responds to the stooges on the chess board. It has not been able to connect to any of its previous experiences and therefore cannot adapt to the planning process. Google Al-phaGo, which prevailed over the individual Go Champion, is yet another example of a reactive method.

Restricted memory consists of ML models that derive comprehension from facts, encrypted knowledge or events that have been learned. With the exception, perhaps, of reactive computers, limited memory benefits from the old days through the observation of events or the information provided to create expertise. Most of the applications known are in the same AI class. Restricted memory systems may retain information for a short period of time. For example, a few cars, chat bots, and virtual private helpers use limited memory innovation.

\section{EMERGENCE OF THE Al}

The world has experienced tremendous industrial advances that have led to the development of AI systems that are currently controlling many sectors, including the financial sector. Steam engine development was the first revolution to take place in 1784 . This was followed by the development of electricity in 1870, which made it possible to use machines. In addition, information technology (IT) came into being in 1969, which led to an emergency AI that made it easier and more accurate to process or process bulk data (Figure 3) (Athey, 2018).

The above revolutions have transformed the concept of Artificial Intelligence into the hypothesis and integration of information systems capable of performing tasks that usually involve human brains, like visual perception, speech identification, decision-making as well as language translation (Baydin et al., 2017). Alan Turing noticed that many people were able to analyze information, solve complicated problems, and make sound decisions when computers were unable to do so. Focused on this, Turing (1950) devised an operating test presented in his journal, "Computing Machinery and Intelligence." Currently, the assessment is often used as a benchmark to assess the computer's ability to function as a normal being. The Turing Test laid the foundation for machine learning, evolutionary computing, and genetic programming.

This paper concentrates on the AI application in the financial sectors. As a result of massive funding from Japan, the United Kingdom and the United States, the very first AI solutions infiltrated the financial sector in 1982, when James Simons set up the quantifiable investment company Renaissance Technologies. Subsequently, they were renowned for innovative approaches to deep learning financial analysis. In the 1990s, AI was involved in the fraudulent financial detection (Mullainathan \& Spiess, 2017). The Financial Crimes Enforcement Network (FINCEN) launched a 
financial crime monitoring technique called the FinCEN Artificial Intelligence System (FIAS) in 1993. The results were outstanding and the machine was able to evaluate more than 200000 financial activities per week and, in about two years, the platform identified 400 tries at $\$ 1$ billion in financial fraud.

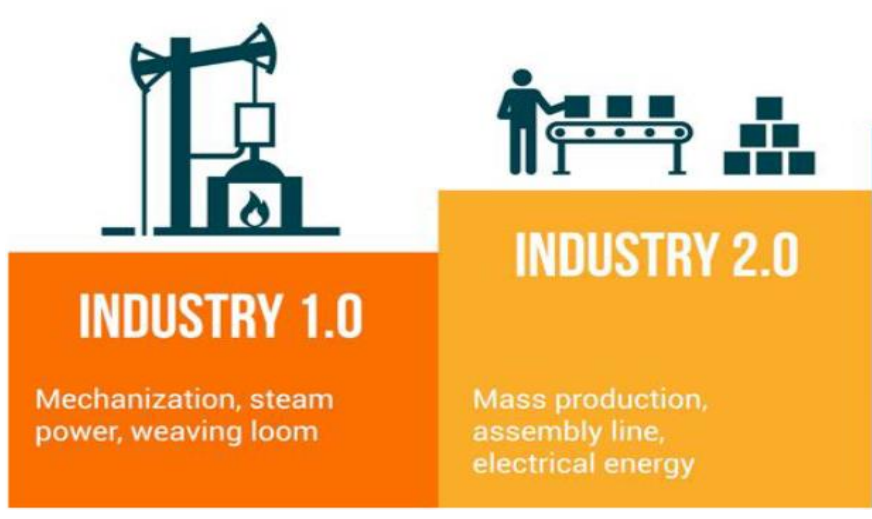

Figure 3: Emergency of AI from other revolutions

\section{ARtificial Intelligence in Changing Financial SERVICES}

The financial sector is one of the main contributors to $\mathrm{Ai}$ technology and its use is growing rapidly. Hedge funds and high-frequency (HFT) financial firms have become AI's first subscribers, but many of the implementations have since entered other parts of the financial sector, including banks, insurance institutions, policy makers and various FinTech platforms. There are a number of diverse AI applications in such enterprises, and the population is rising high. Many of the key aspects include electronic trading, portfolio structure and optimization, robotic consulting services, automated customer services, business forecasting, and other forms of data-intensive analysis. The paper focuses specifically on some of those particular uses where AI is changing the existing conventional financial sector.

\section{Algorithmic Trading}

Algorithmic Trading (AT), also known as the Automatic Trading System, has been one of the biggest participants in the international capital markets. AT traces back to the 1970s, when automated financial systems have been implemented on American capital markets (Begum et al., 2012). Cartea et al (2015) describes AT as applying financial rules to such an advanced system, but then uses them for trading purposes. Currently, AT has put in place a complex AI algorithm that is capable of acting incredibly quickly and is therefore capable of trading much faster than humans. HFT and machines account for 50-70 per cent of all transactions on financial markets and 60 per cent of futures transactions. People usually predict that this figure will be even higher in the US and slightly lower at almost $40 \%$ in developing countries like India.

These systems are important because they have the capacity to carry out transactions at the best possible price. In their presence, a computer often has a significantly reduced chance of making mistakes than a human being. In addition, they have the potential to deal with data loads at once and to track multiple markets at the same time. Finally, interventions do not have an impact on trade or are likely to minimize undue decisions (Kuroda, 2017). Preferably, in order to be able to use AT and HFT, it is necessary to have the least potential timing differences and a high degree of automation and consolidation capacity within the stock market. HTF trade is made up of multinational trading companies spread across multiple treasury bonds, such as equities, corporate bonds, ETFs, and futures. Hedge funds, corporate banking institutions and companies are all recognized for the use of the HTF.

At present, AT and HFT platforms are capable of doing business in milliseconds. For example, the graphs below illustrate the tick-by-tick price swings of E-mini S\&P 500 derivatives (ES) and SPDR S\&P 500 ETFs at separate time intervals. By zooming in the figures, a large price gap between the two stocks is identified. They look completely related at first sight. The more one zooms in the diagrams, the higher the price difference for securities. In this scenario alone, what appears to be ideally linked ends up being lucrative from the point of view of extremely fast AT robotics (Treleaven \& Batrinca, 2017). Such spreads may seem slim, but algorithmic trading platforms are capable of producing large amounts of such small enterprises on a daily basis, with the possibility of making massive profits. 
(d) 250 Milliseconds

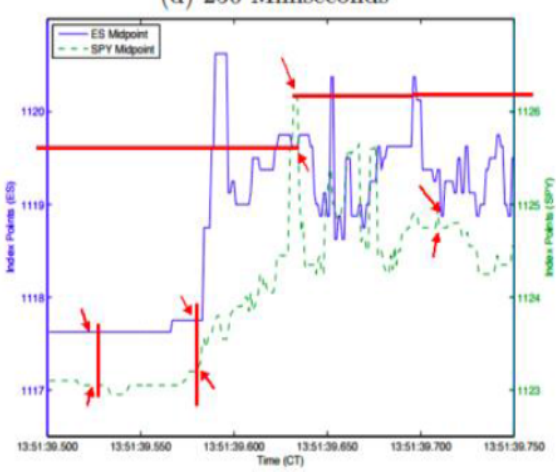

(c) Minute

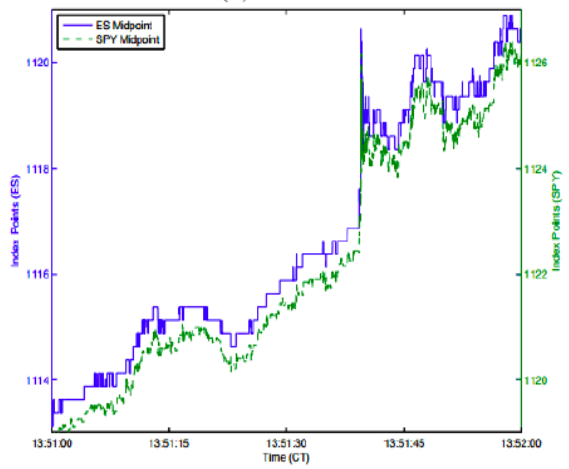

(b) Hour

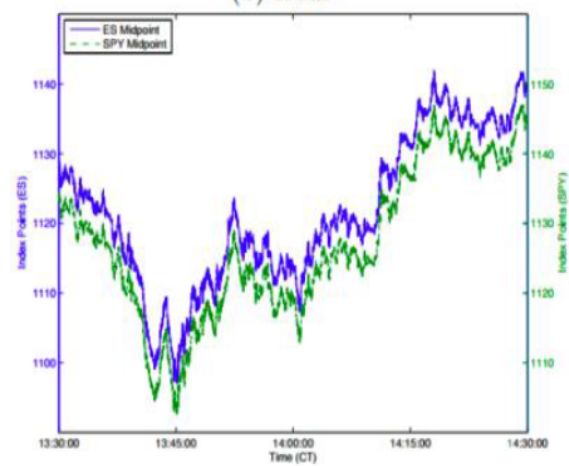

Figure 4: Graph demonstrating Algorithmic trading at distinct time frequencies

\section{Fraud Detection and Compliance}

The money associated with compliance in most finance industry is relatively high to avoid related penalties. For this reason, banks are now introducing AI technology to make such transactions more efficient and reliable. In addition, McAfee reports that cyber-crimes currently cost the financial sector around $\$ 600$ billion annually, although identity fraud has been the most prevalent. A number of financial firms are currently implementing AI to detect fraud. Each one of them is a MasterCard (Ahmed \& Siddique, 2013). Their AI program, called "Decision Intelligence," uses available data to recognize trends in past MasterCard usage in order to create a "default transaction," and then MasterCard contrasts and scores any new clients' transactions versus their generic transactions. Another obstacle to the identification and enforcement of fraud is to minimize transactions too vigorously (Wilson \& Daugherty, 2018). The Javelin Strategy Study (2015) indicated that incorrectly rejected transactions constitute a major challenge for financial services.

\section{Chatbots and Robotic Advisory Services}

A Chatbot is capable of responding to customers instantly and appears to be able to provide 24-hour support 7 days a week, and 64 per cent of people are optimistic about talking to a Chatbot because they are both safer and more efficient than talking to a person. Such Chatbot frameworks are capable of managing the number of users with a high level of user support at a low cost. The Chatbot is centered on NLP and ML algorithms designed to provide a customized conversation framework ( $\mathrm{Lu}$ et al., 2018). Robotic advisory are algorithms designed to identify the best package for a client based on the client's risk preferences and target. Currently, the benefits of using Chatbots have made it an industry practice for financial institutions with a broad customer base, such as banks (Rouhiainen, 2018). Today, the robotic advisory application, the Clerkie - combination of a chatbot with a robotic advisory application that manages the finances of all users using your smartphone (Donepudi, 2018b). Loans from different banks can be used, and the AI engine is able to evaluate the revenue and expenditure of customers in order to predict how much they should invest and save. This has the advantage of accessibility or restructuring of regulatory financial instruments, costeffectiveness in which diversified, low-priced asset allocations and behavioral biases are required. As a result, robotic advisory applications would save up to $4.4 \%$ of costs annually compared to conventional financial advice.

\section{Cyber Security}

Cyber security and data protection are important because they reduce the risk of losing valuable and confidential data, which is why AI is needed to address this vulnerability. AI has infinite scope in many industries and markets. Similarly, the enforcement of a stricter network security scheme with the help of AI might also help businesses secure from cyber-attacks and identify newer viruses (Horowitz et al., 2018). In addition, these models can promote proper safety practices as well as avoid mistakes, making them a no-brainier to implement in a variety of instances. However, it is also important to remain aware that AI Technologies chips could have malicious code and adware configured for a variety of reasons of political concern (Donepudi, 2015). As a result, the deployment of AI has made it possible to use different forms of biometric authentication for even more secure access (Donepudi, 2018a). Some of these AI applications include fingerprint scanners, sensors and palm images, and facial detection. Without even implementing these operations in comparison to the original password, the cyber-criminals' hurdle has increased considerably. All of this underlines the need for consistency and precision in the advancement of AI until innovation is implemented on critical internet platforms.

\section{Accounting and Auditing}

Accounting and auditing are experiencing a radical shift due to advances in data mining and AI. Closely reflecting on the Deep Shift 2015 study of the World Economic 
Forum, 816 senior IT and communications executives participated in the Technology Tipping Points and Social Effects survey. They announced that 75 per cent of participants accepted that a 30 per cent turning point for AI business audits would be reached by 2025 (Abedin et al., 2012). One of the best aspects of $\mathrm{AI}$ is that it is exceptional to match trends, making technology accessible to a wide range of industries and large enterprises (Kokina \& Davenport, 2017). Specifically, the Deep Shift study points out that more workers would soon be replaced by computer algorithms and robotics.

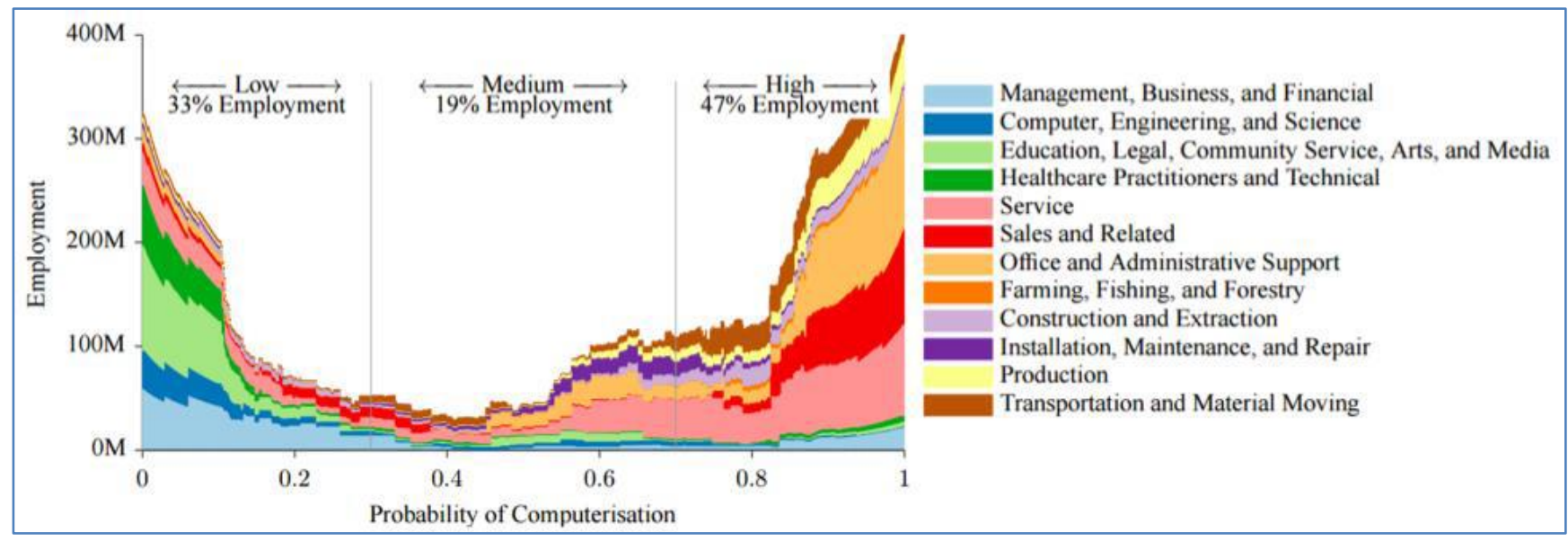

Figure 5: Computerized occupational distribution in the US

Auditing is particularly well suited to AI and business intelligence implementations, as it has become very difficult to incorporate large amounts of both organized and unstructured data in order to gain understanding of the financial and non-financial outcomes of businesses. Furthermore, as many of the auditing activities are organized and redundant, the partnership with AI will simplify these operations. In more basic cases, AI could be used to systematically encode account inputs and to build and enhance fraud detection mechanisms by developing an advanced ML code (Shabbir \& Anwer, 2018). As far as the audit market is concerned, more sophisticated AI technologies such as DL can be used to analyze both multiple data sources in order to compile reports or predict accurate forecasts. The variety of DL data that can be scanned is very broad and can cover everything from social media messages, audio files, conference calls and emails. By introducing AI in accounting and auditing, transactions can be checked more closely by allowing a greater volume of data to be inspected.

\section{Other AI Applications}

The company's plans are very relevant to finance. Automation of company plans allows for a more in-depth assessment of contract terms and valuations. In addition, a quick impression of past performance, asset pricing, risk exposure and projected productivity of contractors can be taken from the proposed trade agreement. This makes it easier for businesses and customers to be much more effective in a process that is often time consuming and complicated. The reduction of additional costs in this region could make the entire business plan more productive than previously expected.

\section{Al Adoption in Financial Sector \& Policy IMPLICATIONS}

Pioneers and developers are currently facing a number of challenges in implementing AI. App developers are struggling to acquire $\mathrm{AI}$ that is known to contribute and recognize usage occurrences. Leader's issues have shifted from 'if' to 'how.' 'Pioneers are working to solve challenges in attracting talent and resolving societal opposition to AI. Financial institutions carry out a wide range of operations, ranging from collecting basic information to making informed decisions or otherwise advising policy makers. At present, financial structure protocols are purposefully designed to leverage the collective mental capacity and comprehension of a number of individuals. Executives should be encouraged to reorganize their processes in order to have full AI capability.

Adequate planning and policy are needed to build and maintain a dynamic environment for AI to support the economy more broadly. The implementation of digital technology is a crucial measure to ensure that companies can compete successfully across different industries and locations. AI is the primary set of approaches to be used for a specific business case. Leaders in the company need to be aware that the concentration of the workforce is increasing, not replacing it. In addition, the provision of knowledge and analytical culture is more important than the AI capability. If the findings and implications are not achieved, neither the predictive nor the descriptive mechanism can be achieved. ML requires you to benefit from the mechanisms of the available datasets. There seem to be a number of exceptions at the staff level to help all 
those involved. The AI project framework will allow decision-making by executives. In-house lifestyle startups can allow users to develop their own strategies to a complex challenge.

Finally, financial institutions must upgrade their IT operating model in order to plan for the new standard. Firms should develop their technology skills, policies and be more intelligent about the needs of their customers. The organization should focus on cyber security before it appears to be urgent. It is important for companies to ensure that they have sole rights to the talents and abilities needed to perform and win.

\section{ConClusion}

The main objective of this paper was to analyze the impact of AI on the digital society, particularly in the financial sector. It also sought to describe the implementation of AI, its constraints, its prospects and its impact on employment and services. For this study, a document on the qualitative review of the process was used to gather information on the implementation and impact of AI, as well as a proposal for the adoption of AI by the banking sector. It is noted that the financial sector may be very keen to design and adopt AI for speed, accuracy and performance. AI makes a significant contribution to changing and changing the culture of work and efficiency, and there is a huge potential. The new surge in AI-based applications aims to be the largest and most influential technological transition in the past. Its general-purpose existence, which facilitates the use of these newest technologies in different sectors and occupations, irrespective of the type of professionalism of employees in the workplace, is a source of widespread collective fear of job losses and the loss of power over the lives of individuals. Every business can use AI technology for financial and accounting purposes. It is important for companies and society as a whole to learn how to use and improve new technologies. In order to remain competitive, firms will need to incorporate $\mathrm{AI}$, and workers will need to adapt their skills in order to retain employment.

\section{REFERENCES}

Abedin, M. M., Ahmed, A. A. A., \& Neogy, T. K. (2012). Mechanism of Accountability and Auditing: Public Sector Scenarios of Bangladesh. Journal of Business Studies, 4, 131-148.

Ahmed, A. A. A., \& Siddique, M. N.-E.-A. (2013). Internet Banking Espousal in Bangladesh: A Probing Study. Engineering International, 1(2), 93-100. https://doi.org/10.18034/ei.v1i2.211

Athey, S. (2018). The impact of machine learning on economics The economics of artificial intelligence: An agenda (pp. 507-547): University of Chicago Press. https://scholar.google.com/scholar?hl=en\&as sdt=0\%2C5 \&as_ylo=2010\&as_yhi $=2022 \& q=T h e+i m p a c t+o f+$ machine +
learning+on+economics+The+economics+of+artificial+inte lligence $\% 3 \mathrm{~A}+\mathrm{An}+$ agenda\&btnG $=$

Baydin, A. G., Pearlmutter, B. A., Radul, A. A., and Siskind, J. M. (2017). Automatic differentiation in machine learning: a survey. The Journal of Machine Learning Research, 18(1), 55955637. https://dl.acm.org/doi/abs/10.5555/3122009.3242010

Begum, R., Ahmed, A. A. A., \& Neogy. T. K. (2012). Management Decisions and Univariate Analysis: Effects on Corporate Governance in Bangladesh. Journal of Business Studies, 3, 87-115.

Cartea, A., S. Jaimungal, and J. Penalva (2015). ' Algorithmic and High-Frequency Trading (1st ed.). Cambridge: Cambridge University Press.

Chui, M., Manyika, J., and Miremadi, M. (2015). Four fundamentals of workplace automation. McKinsey Quarterly, 29(3), 1-9. https://roubler.com/sg/wpcontent/uploads/sites/49/2016/11/Four-fundamentalsof-workplace-automation.pdf

Chui, M., Manyika, J., and Miremadi, M. (2016). Where machines could replace humans-and where they can't (yet). McKinsey Quarterly, 30(2), 1-9. http://pinguet.free.fr/wheremachines.pdf

De Prado, M. L. (2018). Advances in financial machine learning: John Wiley \& Sons. https:/ / books.google.com/books?hl=en\&lr=\&id=oU9KD wAAQBAJ\&oi=fnd\&pg=PR21\&dq=Advances+in+financial +machine+learning:+John+Wiley+\%26+Sons\&ots=7UDHV Axey\&sig=10ymGgObxzI6ZUrk3XYPY1EVXD8

Donepudi, P. K. (2015). Crossing Point of Artificial Intelligence in Cybersecurity. American Journal of Trade and Policy, 2(3), 121-128. https://doi.org/10.18034/ajtp.v2i3.493

Donepudi, P. K. (2018a). AI and Machine Learning in Retail Pharmacy: Systematic Review of Related Literature. $A B C$ Journal of Advanced Research,7(2), 109-112. https://doi.org/10.18034/abcjar.v7i2.514

Donepudi, P. K. (2018b). Application of Artificial Intelligence in Automation Industry. Asian Journal of Applied Science and Engineering, 7(1), 7-20. http://doi.org/10.5281/zenodo.4146232

Gomber, P., Kauffman, R. J., Parker, C., and Weber, B. W. (2018). On the fintech revolution: Interpreting the forces of innovation, disruption, and transformation in financial services. Journal of Management Information Systems, 35(1), 220-265. https://doi.org/10.1080/07421222.2018.1440766

Horowitz, M. C., Allen, G. C., Saravalle, E., Cho, A., Frederick, K., and Scharre, P. (2018). Artificial intelligence and international security: Center for a New American Security. http://www.indexfunds.org/resources/ResearchMaterials/NatSec/Strategic Competition_in Era of AI.pdf

Kokina, J., and Davenport, T. H. (2017). The emergence of artificial intelligence: How automation is changing auditing. Journal of Emerging Technologies in Accounting, 14(1), 115-122. https://meridian.allenpress.com/jeta/articleabstract $/ 14 / 1 / 115 / 116001$

Kuroda, H. (2017). AI and the Frontiers of Finance. Paper presented at the Speech given by the Governor of the Bank of Japan at the Conference on "AI and Financial Services/Financial Markets. https://pdfs.semanticscholar.org/cc08/475f1efd0e74701e0 18362fba8cd302e8511.pdf 
Lu, H., Li, Y., Chen, M., Kim, H., and Serikawa, S. (2018). Brain intelligence: go beyond artificial intelligence. Mobile Networks and Applications, 23(2), 368-375. https://link.springer.com/article/10.1007/s11036-017-0932-8

Mackenzie, A. (2015). The production of prediction: What does machine learning want? European Journal of Cultural Studies, 18(4-5), https://doi.org/10.1177\%2F1367549415577384

Mullainathan, S., and Spiess, J. (2017). Machine learning: an applied econometric approach. Journal of Economic Perspectives, 31(2), $87-106$. https://www.aeaweb.org/articles?id=10.1257/jep.31.2.87

Rouhiainen, L. (2018). Artificial Intelligence: 101 things you must know today about our future: Lasse Rouhiainen. https://books.google.com/books?hl=en\&lr=\&id=P3fSDw AAQBAI\&oi=fnd\&pg=PP1\&dq=Artificial+Intelligence: +10 $1+$ things + you +must+know+today+about+our+future\&ots =TZYjzBx0tq\&sig=Fzfr8YbzD5m8g0s9Rmx2jwy4ZuU

Shabbir, J., and Anwer, T. (2018). Artificial intelligence and its role in near future. arXiv preprint arXiv:1804.01396. https://arxiv.org/abs/1804.01396
Sharma, R., Mithas, S., and Kankanhalli, A. (2014). Transforming decision-making processes: a research agenda for understanding the impact of business analytics on organisations: Taylor \& Francis. https://orsociety.tandfonline.com/doi/abs/10.1057/ejis.2 $\underline{014.17}$

Treleaven, P., and Batrinca, B. (2017). Algorithmic regulation: automating financial compliance monitoring and regulation using AI and blockchain. Journal of Financial Transformation, 45 , 14-21. https:/ / files.openpdfs.org/jE1d4GBZ5Ob.pdf\#page=14

Turing, A. M., (1950). Computing Machinery and Intelligence, Mind59: 433-460; reprinted in (Copeland, 2004)

Wilson, H. J., and Daugherty, P. R. (2018). Collaborative intelligence: humans and $\mathrm{AI}$ are joining forces. Harvard Business Review, 96(4), 114-123. https://www.accenture.com/t00010101t000000z_w____acnm edia/pdf-84/accenture-collaborative-intelligence-2018.pdf 


\section{How to Cite This Article?}

Donepudi, P. K. (2019). Automation and Machine Learning in Transforming the Financial Industry. Asian Business Review, 9(3), 129-138. https://doi.org/10.18034/abr.v9i3.494

Online Archive: https://abc.us.org/ojs/index.php/abr/issue/archive 